with recent asthma symptoms (Kendall's Tau-B $=0.236, \mathrm{p}=$ 0.016).

Serum periostin levels significantly correlated with asthma symptoms during the last 12 months in 10-year-old children who were infected with RSV during their first two years of life. Our preliminary results suggest that serum periostin level may be useful in diagnosing asthma in children, especially those with recent asthma symptoms and history of RSV infection in infancy.

\section{ORAL BETA-LACTAM CHALLENGE TEST WITHOUT ANTECEDENT SKIN TESTING IN CHILDREN WITH HISTORY OF ALLERGY TO BETA-LACTAM}

Iva Mihatov Štefanović* Renata Vrsalović, Gabrijela Perić Marković. University Hospital Center Sestre Milosrdnice

\subsection{6/archdischild-2021-europaediatrics. 165}

Beta-lactam (BL) antibiotics are among the most prescribed drugs globally but can provoke hypersensitivity reactions which can lead to incorrectly labelling as 'allergy'. Data on prevalence and incidence of drug hypersensitivity reactions (DHRs) are limited, especially in the pediatric age. It is important to assess subjects with history of hypersensitivity reactions to $\mathrm{BL}$ as up to $70 \%$ are not allergic based on diagnostic test results. Laboratory tests for identifying children who are allergic to drugs have low diagnostic accuracy and predictive value. The gold standard to diagnose DHR is drug provocation test (DPT). DHRs are classified as immediate or nonimmediate/delayed reactions. Mild delayed cutaneous reactions including maculopapular rashes and urticaria/angioedema are common reactions in children and often occur in the setting of viral infections. In mild cutaneous delayed reactions, some clinicians suggest performing only DPT because of its high negative predictive value, without skin testing and serum IgE measurement while other suggest SPT and/or specific IgE to culprit drug if patient/caregiver cannot provide a detailed description of the previous reaction.

Aim: to investigate the proportion of positive oral provocation tests in children labeled as BL antibiotic allergy.

Direct oral challenge was performed in patients with history of benign rash associated with beta-lactam antibiotic. Because detailed description of suspected allergic reaction could not be provided from all caregivers, all patients went specific $\operatorname{IgE}$ to amoxicillin and/or basophil degranulation test to culprit drug which was negative. 19 patients were included, average age 6 years and 1 month. In 17 patients suspected allergic reaction was caused by amoxicillin (AMX); 8 of which in combination with clavulanic acid, one with phenoxymethylpenicillin and one with cephalexin. A DPT involved open 3step graded oral provocation test with AMX, time interval between the doses was $2 \mathrm{~h}$. The patients were monitored for $24 \mathrm{~h}$ after challenge and were discharged with instructions to call in the event of a delayed reaction.

After DPT there was no reaction in $89 \%$ of patients, one patient developed benign rash and one drug-induced enterocolitis syndrome.

BL antibiotics can be safely readministered to children with history of benign rash to beta-lactams using graded oral challenge in medically supervised settings if $\operatorname{spIgE}$ is negative even if uncertain history. It is important to establish a correct diagnosis of BL antibiotic allergy since using alternative non- $\beta$-lactam antibiotics in these patients leads to higher healthcare costs and adverse events.

\section{EFFICACY OF DUPILUMAB IN CHILDREN WITH UNCONTROLLED, MODERATE-TO-SEVERE TYPE 2 ASTHMA, WITH AND WITHOUT EVIDENCE OF ALLERGY ENROLLED IN PHASE 3 VOYAGE}

Stanley J Szefler, Leonard B Bacharier, Jorge F Maspero, Nikos Papadopoulos, Christian Domingo*, Nadia Daizadeh, David J Lederer, Megan Hardin, Juby A Jacob-Nara, Yamo Deniz, Rebecca Gall, Benjamin Ortiz, Michel Djandji, Paul J Rowe. Royal Manchester Children's Hospital, Manchester, UK; 2nd Pediatric Clinic, University of Athens, Greece

\subsection{6/archdischild-2021-europaediatrics. 166}

The allergic phenotype is an important subset of asthma patients with type 2 inflammation. Dupilumab, a fully human monoclonal antibody, blocks the shared receptor component for IL-4/IL-13, key and central drivers of type 2 inflammation in multiple diseases. In phase 3 VOYAGE, add-on dupilumab $100 \mathrm{mg} / 200 \mathrm{mg}$ (body weight $\leq 30 \mathrm{~kg} />30 \mathrm{~kg}$, respectively) every 2 weeks vs placebo, reduced severe asthma exacerbations by $59.3 \% \quad(\mathrm{P}<0.0001)$ and improved lung function in children aged 6 to $<12$ years with uncontrolled moderate-to-severe type 2 asthma (baseline blood eosinophils $\geq 150$ cells $/ \mu \mathrm{l}$ or FeNO $\geq 20 \mathrm{ppb}$ ). This analysis evaluated the efficacy of dupilumab in pediatric patients with type 2 asthma with/without evidence of allergic asthma (total serum $\operatorname{IgE} \geq 30 \mathrm{IU} / \mathrm{mL}$ and $\geq 1$ perennial aeroallergen-specific $\mathrm{IgE} \geq 0.35 \mathrm{kU} / \mathrm{L}$ at baseline).

Annualized severe exacerbation rate during the 52-week treatment period was assessed using a negative binomial model.

350 pediatric patients with type 2 asthma were enrolled: of which, 261 had evidence of allergic asthma and 89 did not. Baseline characteristics were similar between subgroups, except for prevalence of ongoing atopic comorbidities $(99.6 \%$ vs $77.5 \%$ ) and median levels of type 2 biomarkers (blood eosinophils: 540.00 cells $/ \mu \mathrm{L}$ vs 310.00 cells $/ \mu \mathrm{L} ;$ FeNO: $28 \mathrm{ppb}$ vs $13 \mathrm{ppb}$; total serum IgE: $657.00 \mathrm{IU} / \mathrm{mL}$ vs $107.00 \mathrm{IU} / \mathrm{mL}$ ) which were all higher in patients with vs without evidence of allergic asthma. Dupilumab vs placebo significantly reduced annualized severe exacerbation rate by $62 \%(\mathrm{P}<0.0001)$ in patients with, and $51 \%(\mathrm{P}<0.05)$ in patients without evidence of allergic asthma. No significant interaction was observed between the treatment effect and evidence of allergic asthma. In the overall safety population, the incidence of treatment-emergent adverse events (TEAEs) was similar across treatment groups; the most common TEAE occurring more frequently in the dupilumab group was injection site erythema (12.9\% dupilumab vs $9.7 \%$ placebo).

The majority of pediatric type 2 asthma patients enrolled in VOYAGE had evidence of allergic asthma; these patients had very high levels of type 2 biomarkers. Dupilumab demonstrated efficacy in reducing severe asthma exacerbations in children aged 6 to $<12$ years with uncontrolled, moderate-tosevere type 2 asthma, with or without evidence of allergic asthma. 